\title{
Ein Veranstaltungspaket
}

TheraPro 2022 Stellen Sie sich am 29. und 30. Januar 2022 in Stuttgart Ihr persönliches Veranstaltungspaket zusammen - Sie haben die Wahl: Klientenzentrierung und Selbstfürsorge auf dem ergotag, Covid-19 und Neurorehabilitation auf dem neuroreha-Tag sowie eine interprofessionelle Podiumsdiskussion zum Thema Schmerz. Parallel dazu besuchen Sie die Therapiemesse TheraPro.

\section{neuroreha-Tag}

Samstag, den 29. Januar 2022

09:00-10:30 Uhr

Coronavirus-Pandemie - Herausforderung für die Neuroreha

Prof. Dr. med. Thomas Platz, Neurologe

Auswirkungen des coronabedingten Lockdowns auf Menschen nach Schlaganfall - eine retrospektive Studie

Dr. Jeremia Held, Physiotherapeut

App-gestützte Therapieverfahren im ambulanten Setting

Prof. Dr. Martin Lotze, Neurologe und Hirnforscher

11:15-12:45 Uhr

Physiotherapeutisches Assessment für die neurologische Frührehabilitation

Jana Stürner, Physiotherapeutin

Münchner Parkinson Netzwerk Therapie (PaNTHer) -

ein Modellvorhaben für eine bessere sektorenübergreifende Versorgung

Kerstin Ziegler, Physiotherapeutin

Ambulante Schlaganfallnachsorge: Bezugstherapeut*innen lotsen durch die Nachsorge

Maike Bamberger, Ergotherapeutin, und Claudia Pott, MSc, Physiotherapeutin

14:15-15:45 Uhr

Schwindel bei neurologischen Patienten in der ambulanten Versorgung

Ann Kathrin Saul, Physiotherapeutin

Spiegeltherapie in der ambulanten Physio- und Ergotherapie -

wissen, wem's hilft

Prof. Dr. Holm Thieme, Physiotherapeut

Alle Infos zum neuroreha-Tag: www.ergotag.de > "neuroreha-Tag“ 


\section{ergotag}

Sonntag, den 30. Januar 2022

09:45-10:45 Uhr

Selbstfürsorge für Therapeut*innen

Karin Probst

10:45-11:45 Uhr

Coaching als Ressource für Klient und Therapeutin Sara Hiebl

13:00-13:45 Uhr

In zwei Welten - Unterstützung beim Leben mit Autismus

Christine Preißmann

13:45-14:30 Uhr

Handeln gegen Trägheit

Andreas Pfeiffer

15:00-16:00 Uhr

Wo fängt Klientenzentrierung an und wo hört sie auf?

Michael Schiewack, Robert Striesow

Alle Infos zum ergotag: www.ergotag.de

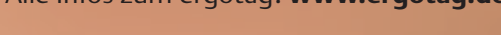

Freitag, 28. bis Sonntag, 30.1.2022

Die Aussteller der TheraPro freuen sich auf Sie, stehen für Fragen und Gespräche zur Verfügung und bieten Workshops und Vorträge an. Mit Ihrer Karte für den ergotag und/oder neuroreha-Tag erhalten Sie an allen drei Tagen kostenfreien Eintritt zur Fachmesse.

Mehr Infos unter

www.messe-stuttgart.de/therapro

\section{Team works!}

Samstag, den 29. Januar 2022

16:30-18:00 Uhr

Schmerztherapie im ambulanten Setting: Wie kann eine optimale Patientenversorgung gelingen?

Interprofessionelle Podiumsdiskussion mit Berufsangehörigen aus Ergotherapie,

Physiotherapie und Medizin

Die Teilnahme ist kostenfrei, um Anmeldung wird gebeten.

Alle Infos zur Podiumsdiskussion:

www.ergotag.de > „Podiumsdiskussion“

\section{Bitte beachten Sie}

In der nach wie vor dynamischen Pandemielage gehen wir zum Zeitpunkt der Drucklegung davon aus, dass Ende Januar 2022 in Baden-Württemberg die Alarmstufe gilt. Damit wäre die Teilnahme am ergotag lediglich immunisierten Personen, also geimpften und/oder genesenen, möglich (2G). Die aktuellen Bestimmungen finden Sie unter www.messe-stuttgart.de/therapro/ besucher/aktuelles/sicherer-messebesuch/. 